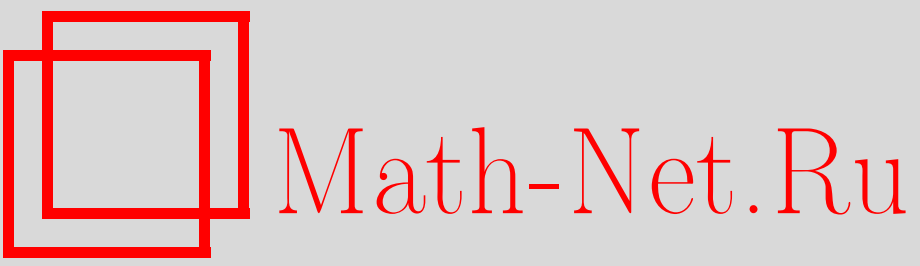

А. В. Латышев, А. А. Юшканов, Метод сингулярных уравнений в граничных задачах кинетической теории, ТМФ, 2005, том 143, номер 3, 437-454

DOI: https://doi.org/10.4213/tmf1824

Использование Общероссийского математического портала Math-Net.Ru подразумевает, что вы прочитали и согласны с пользовательским соглашением

http://www . mathnet.ru/rus/agreement

Параметры загрузки:

IP : 54.162 .85 .209

26 апреля 2023 г., 12:30:08

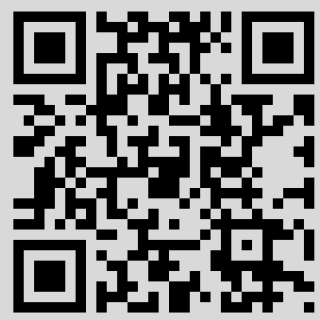


ТЕОРЕТИЧЕСКАЯ

И МАТЕМАТИЧЕСКАЯ

ФИЗИКА

Том 143, № 3

июнь, 2005

(C) 2005 r.

А.В. Латышев*, А. А. Юшканов*

\section{МЕТОД СИНГУЛЯРНЫХ УРАВНЕНИЙ В ГРАНИЧНЫХ ЗАДАЧАХ КИНЕТИЧЕСКОЙ ТЕОРИИ}

Развивается новый эффективный метод решения граничных задач кинетической теории. Метод позволяет получить решение граничных задач для зеркально-диффузных граничных условий с произвольной степенью точности. В основе метода лежит идея разбиения задачи на две, одна из которых имеет диффузное условие отражения молекул от стенки, а вторая - зеркальное условие. Метод излагается на примере классических задач кинетической теории - задачи Крамерса (об изотермическом скольжении) и задачи о тепловом скольжении. Используются уравнение Бхатнагара-Гросса-Крука (с постоянной частотой столкновений) и уравнение Вильямса (с частотой столкновений, пропорциональной скорости молекул).

Ключевые слова: граничная задача, кинетическое уравнение, задача Крамерса, задача о тепловом скольжении, коэффициенты изотермического и теплового скольжений.

\section{1. ВВЕДЕНИЕ}

Метод сингулярных интегральных уравнений является важным методом в теоретической и математической физике, с помощью которого был решен ряд значительных проблем [1]. В кинетической теории точному аналитическому решению поддается сравнительно узкий класс граничных задач [2], [3]. Поэтому развитие новых эффективных методов в этом направлении является актуальной проблемой.

К настоящему времени имеется ряд граничных задач кинетической теории, решенных аналитически (см., например, [4]-[6]). Однако в этих задачах условие на стенке является либо диффузным, либо почти зеркальным. Если взять в этих задачах более общее граничное условие, например, зеркально-диффузное условие (условие Максвелла) на стенке, точное решение построить не представляется возможным.

В то же время ошушается настоятельная потребность в решении ряда задач как для электронов в металле (скин-эффект), так и для разреженных газов (скольжение и скачки макропараметров на границе газ-твердое тело) при наличии общих зеркально-диффузных граничных условий.

* Московский государственный областной университет, Москва, Россия. E-mail: avlatyshev@comail.ru, yushkanov@mtu-net.ru 
В связи с этим в данной работе развивается метод, позволяющий получить решение задачи с зеркально-диффузным граничным условием Максвелла с произвольной степенью точности.

Метод основан на использовании сингулярных интегральных уравнений с ядром Коши и на точном решении задачи с диффузным условием и задачи с зеркальным условием на стенке.

В работе [7] также был предложен метод для решения граничных задач с условием Максвелла, основанный на приближенном решении сингулярных интегральных уравнений. Особенностью предлагаемого в данной работе метода, в отличие от рассмотренного в работе [7], является совпадение с точными результатами при $q \rightarrow 0$ и при $q=1$, т.е. когда коэффищиент аккомодации тангенциального импульса принимает предельные значения: $q=0$ соответствует чисто зеркальному рассеянию молекул на стенке, $q=1$ - полностью диффузному рассеянию.

Полученные в работе результаты показывают, что в случае постоянной частоты столкновений в задаче Крамерса значения коэффициента скольжения отличаются от значений того же коэффищиента, рассчитанного в работе [8] на основе применения численных методов решения кинетического уравнения: при $q=0.1-$ на $0.014 \%$, при $q=0.5$ - на $0.034 \%$, при $q=0.9$ - на $0.087 \%$; при $q=1$ результаты совпадают. В задаче о тепловом скольжении эти значения отличаются при $q=0.1$ на $0.015 \%$, при $q=0.5$ на $0.028 \%$, при $q=0.9$ на $0.006 \%$; при $q=1$ значения совпадают.

\section{2. УРАВНЕНИЕ БХАТНАГАРА-ГРОССА-КРУКА}

Как известно [2], [5], [7], задача Крамерса с условием Максвелла на стенке сводится к решению следующей граничной задачи:

$$
\begin{gathered}
\mu \frac{\partial \psi}{\partial x}+\psi(x, \mu)=\frac{1}{\sqrt{\pi}} \int_{-\infty}^{\infty} e^{-\mu^{\prime 2}} \psi\left(x, \mu^{\prime}\right) d \mu^{\prime}, \\
\psi(0, \mu)=(1-q) \psi(0,-\mu), \quad \mu>0, \\
\psi(x, \mu)=\psi_{\text {as }}(x, \mu)+o(1), \quad x \rightarrow+\infty, \quad \mu<0,
\end{gathered}
$$

где

$$
\psi_{\text {as }}(x, \mu)=2 U_{0}+2 g_{v}(x-\mu),
$$

$U_{0}$ - неизвестная скорость изотермического скольжения, $g_{v}$ - градиент безразмерной массовой скорости.

Разделение переменных в уравнении (2.1) приводит к выражению

$$
\psi_{\eta}(x, \mu)=e^{-x / \eta} \Phi(\eta, \mu),
$$

где $\eta$ - спектральный параметр, или параметр разделения.

Подставляя (2.4) в (2.1), приходим к характеристическому уравнению

$$
(\eta-\mu) \Phi(\eta, \mu)=\frac{1}{\sqrt{\pi}} \eta
$$


с условием (нормировкой на собственную функцию $\Phi)$

$$
\int_{-\infty}^{\infty} e^{-\mu^{2}} \Phi(\eta, \mu) d \mu=1
$$

При $\eta \in(-\infty, \infty)$ из уравнений $(2.5),(2.6)$ в пространстве обобщенных функций [9] находим собственные функции непрерывного спектра:

$$
\Phi(\eta, \mu)=\frac{1}{\sqrt{\pi}} \eta P \frac{1}{\eta-\mu}+e^{\eta^{2}} \lambda(\eta) \delta(\eta-\mu) .
$$

Здесь $\lambda(z)$ - дисперсионная функция Черчиньяни, $\delta(x)$ - дельта-функция Дирака, символ $P x^{-1}$ означает главное значение интеграла от $x^{-1}$,

$$
\lambda(z)=1+z \frac{1}{\sqrt{\pi}} \int_{-\infty}^{\infty} \frac{e^{-\tau^{2}} d \tau}{\tau-z} .
$$

Решение задачи (2.1)-(2.3) теперь будем искать в виде

$$
\psi(x, \mu)=\psi_{\text {as }}(x, \mu)+\psi_{\mathrm{c}}(x, \mu),
$$

где

$$
\psi_{\mathrm{c}}(x, \mu)=\int_{0}^{\infty} e^{-x / \eta} \Phi(\eta, \mu) a(\eta) d \eta,
$$

$a(\eta)$ - неизвестная функция (коэффициент непрерывного спектра), функция $\Phi$ определяется равенством $(2.7)$, функции $\psi_{\mathrm{as}}$ и $\psi_{\mathrm{c}}$ являются решениями уравнения $(2.1)$.

С помошью (2.8) перепишем условие (2.2) в виде

$$
\psi_{\mathrm{c}}(0, \mu)-(1-q) \psi_{\mathrm{c}}(0,-\mu)=\psi_{0}(\mu), \quad \mu>0,
$$

где

$$
\psi_{0}(\mu)=(1-q) \psi_{\text {as }}(0,-\mu)-\psi_{\text {as }}(0, \mu)=-2 q U_{0}+2 g_{v}(2-q) \mu
$$

а условие (2.3) - в виде

$$
\psi_{\mathrm{c}}(+\infty, \mu)=0, \quad \mu<0 \text {. }
$$

Воспользовавшись тождеством

$$
A=q A+(1-q) A,
$$

представим $\psi_{\mathrm{c}}(0, \mu)$ и $\psi_{0}(\mu)$ из уравнения (2.10) в виде (2.12). Тогда условие $(2.10)$ можно записать в виде

$$
q \psi_{\mathrm{c}}(0, \mu)+(1-q)\left[\psi_{\mathrm{c}}(0, \mu)-\psi_{\mathrm{c}}(0,-\mu)\right]=q \psi_{0}(\mu)+(1-q) \psi_{0}(\mu), \quad \mu>0 .
$$


Величины $q$ и $1-q$ являются линейно независимыми. Следовательно, условие (2.13) эквивалентно двум условиям:

$$
\begin{array}{ll}
\psi_{\mathrm{c}}(0, \mu)=\psi_{0}(\mu)+(1-q) C(\mu), & \mu>0 \\
\psi_{\mathrm{c}}(0, \mu)-\psi_{\mathrm{c}}(0,-\mu)=\psi_{0}(\mu)-q C(\mu), & \mu>0
\end{array}
$$

где $C(\mu)$ - некоторая неизвестная функция.

Таким образом, задача Крамерса с условием Максвелла на стенке эквивалентна двум граничным задачам, первая из которых состоит из уравнения (2.1), условия (2.11) вдали от стенки и диффузного условия (2.14); вторая задача состоит из уравнения (2.1), условия (2.11) вдали от стенки и зеркального условия (2.15).

Обозначим через $\psi_{\mathrm{c}}^{(1)}(x, \mu)$ и $\psi_{\mathrm{c}}^{(2)}(x, \mu)$ соответственно решения первой и второй граничных задач. Их решение позволяет определить функции $\psi_{\mathrm{c}}^{(1)}(0, \mu)$ и $\psi_{\mathrm{c}}^{(2)}(0, \mu)$ через неизвестную функцию $C(\mu)$. Обозначим также $\psi_{\mathrm{c}}^{a}(\mu)=\psi_{\mathrm{c}}^{(2)}(0, \mu)-\psi_{\mathrm{c}}^{(2)}(0,-\mu)$. Так как в точном аналитическом решении $\psi_{\mathrm{c}}^{(1)}(x, \mu) \equiv \psi_{\mathrm{c}}^{(2)}(x, \mu)$, то очевидно, что функция $C(\mu)$ определяется из условия

$$
\psi_{\mathrm{c}}^{a}(\mu)=\psi_{\mathrm{c}}^{(1)}(0, \mu)-\psi_{\mathrm{c}}^{(1)}(0,-\mu)
$$

Перейдем к решению этих задач.

\section{3. ЗАДАЧА С ДИФФУЗНЫМ ГРАНИЧНЫМ УСЛОВИЕМ}

Начнем с первой задачи. Ищем решение в виде разложения (2.9). Подставляя (2.9) в левую часть условия (2.14), получаем сингулярное интегральное уравнение с ядром Коши:

$$
\frac{1}{\sqrt{\pi}} \int_{0}^{\infty} \frac{\eta a(\eta) d \eta}{\eta-\mu}+e^{\mu^{2}} \lambda(\mu) a(\mu)=\psi_{0}(\mu)+(1-q) C(\mu), \quad \mu>0
$$

Введем вспомогательную функцию

$$
N(z)=\frac{1}{\sqrt{\pi}} \int_{0}^{\infty} \frac{\eta a(\eta) d \eta}{\eta-z}
$$

аналитическую везде в комплексной плоскости кроме разреза вдоль положительной действительной полуоси. Граничные значения этой функции сверху $N^{+}(\mu)$ и снизу $N^{-}(\mu)$ на полуоси $\mu>0$ связаны формулой Сохоцкого:

$$
N^{+}(\mu)-N^{-}(\mu)=2 \sqrt{\pi} i \mu a(\mu), \quad \mu>0 .
$$

Будем считать, что функция $C(\mu)$ аналитически продолжима с действительной полуоси в комплексную плоскость. С помощью граничных значений $N(z)$ и $\lambda(z)$ сведем уравнение (3.1) к краевой задаче:

$$
\begin{aligned}
& \lambda^{+}(\mu)\left[N^{+}(\mu)-\psi_{0}(\mu)-(1-q) C(\mu)\right]= \\
& =\lambda^{-}(\mu)\left[N^{-}(\mu)-\psi_{0}(\mu)-(1-q) C(\mu)\right], \quad \mu>0 .
\end{aligned}
$$


Индекс коэффициента $G(\mu)=\lambda^{-}(\mu) / \lambda^{+}(\mu)$ этой задачи равен $\varkappa(G)=-1$. В самом деле, обозначим через $\theta(\mu)=\arg \lambda^{+}(\mu)$ регулярную однозначную ветвь аргумента, фиксированную в нуле условием $\theta(0)=0$. Заметим, что $\theta(+\infty)=\pi$. Следовательно,

$$
\varkappa(G)=\frac{1}{2 \pi}[\arg G(\mu)]_{(0,+\infty)}=-\frac{1}{\pi}[\theta(\mu)]_{(0,+\infty)}=-\frac{\theta(+\infty)-\theta(0)}{\pi}=-1 .
$$

Рассмотрим однородную задачу, соответствуюшую задаче (3.4):

$$
\frac{X^{+}(\mu)}{X^{-}(\mu)}=\frac{\lambda^{+}(\mu)}{\lambda^{-}(\mu)}, \quad \mu>0 .
$$

Решение задачи (3.5) [10] имеет вид

$$
X(z)=\frac{1}{z} e^{V(z)}, \quad V(z)=\frac{1}{\pi} \int_{0}^{\infty} \frac{\zeta(\tau) d \tau}{\tau-z}, \quad \zeta(\tau)=-\frac{\pi}{2}-\operatorname{arctg} \frac{\lambda(\tau)}{\sqrt{\pi} \tau e^{-\tau^{2}}} .
$$

Преобразуем задачу (3.4) с помощью (3.5) к задаче определения аналитической функции по ее нулевому скачку на разрезе:

$$
\begin{aligned}
X^{+}(\mu)\left[N^{+}(\mu)-\psi_{0}(\mu)-(1-q) C(\mu)\right]= \\
=X^{-}(\mu)\left[N^{-}(\mu)-\psi_{0}(\mu)-(1-q) C(\mu)\right], \quad \mu>0 .
\end{aligned}
$$

Общее решение задачи (3.6) зависит от порядка роста функции $C(\mu)$ в бесконечно удаленной точке. Ограничимся далее для определенности приближениями нулевого и первого порядков, взяв в качестве функции $C(\mu)$ полином первой степени $C(\mu)=C_{0}+$ $C_{1} \mu$ (в нулевом приближении $\left.C(\mu)=C_{0}, C_{1}=0\right)$. Тогда общее решение задачи (3.6) дается формулой

$$
N(z)=-2 U_{0} q+2(2-q) g_{v} z+\left(C_{0}+C_{1} z\right)(1-q)+\frac{d_{0}}{X(z)},
$$

где $d_{0}-$ произвольная постоянная.

Неизвестная функция $a(\eta)$ находится из равенства $(3.3)$, если в него подставить решение (3.7). Функция $N(z)$, введенная равенством (3.2), исчезает в бесконечно удаленной точке, в то время как функция $N(z)$, определенная равенством $(3.7)$, имеет в бесконечно удаленной точке полюс первого порядка. Этот полюс устраняется условием $d_{0}=-2(2-q) g_{v}-(1-q) C_{1}$.

Заметим, что

где

$$
\frac{1}{X(z)}=z-V_{1}+\frac{V_{1}^{*}}{z}+\frac{V_{2}^{*}}{z^{2}}+\cdots, \quad z \rightarrow \infty
$$

$$
\begin{gathered}
V_{k}=-\frac{1}{\pi} \int_{0}^{\infty} \zeta(\tau) \tau^{k-1} d \tau, \quad V_{k}^{*}=\frac{1}{\pi} \int_{0}^{\infty} \frac{\sin \zeta(\tau)}{X(\tau)} \tau^{k-1} d \tau, \quad k=1,2, \ldots, \\
V_{1}=1.016191, \quad V_{2}=0.75, \quad V_{2}^{*}=-0.293606, \\
V_{1}^{*}=-V_{2}+\frac{1}{2} V_{1}^{2}=-0.233678 .
\end{gathered}
$$

Подставим (3.8) в (3.7). Из условия $N(\infty)=0$ получаем уравнение

$$
-2 U_{0} q+(1-q)\left(C_{0}+C_{1} V_{1}\right)+2(2-q) V_{1} g_{v}=0 .
$$

Для нахождения скорости скольжения $U_{0}$ требуются еше два уравнения. Выведем их из решения второй граничной задачи. 


\section{4. ЗАДАЧА С ЗЕРКАЛЬНЫМ ГРАНИЧНЫМ УСЛОВИЕМ}

Решение этой задачи также ишем в виде разложения по собственным функциям характеристического уравнения:

$$
\psi_{\mathrm{c}}(x, \mu)=\int_{0}^{\infty} e^{-x / \eta} \Phi(\eta, \mu) b(\eta) d \eta,
$$

где $b(\eta)$ - неизвестная функция (коэффициент непрерывного спектра).

Подставляя (4.1) в (2.15), приходим к интегральному уравнению

$$
\int_{0}^{\infty} \Phi(\eta, \mu) b(\eta) d \eta-\int_{0}^{\infty} \Phi(\eta,-\mu) b(\eta) d \eta=\psi_{0}(\mu)-q C(\mu), \quad \mu>0 .
$$

Для решения этого уравнения, содержашего интеграл по положительной полуоси и определенного также на положительной полуоси, разовьем специальный метод. Заметим, что левая часть уравнения (4.2) - нечетная функция. Продолжим функцию $b(\eta)$ на отрицательную полуось, полагая

$$
b(-\eta)=-b(\eta), \quad \eta>0 .
$$

Теперь уравнение (4.2) можно преобразовать к следующему виду:

$$
\int_{-\infty}^{\infty} \Phi(\eta, \mu) b(\eta) d \eta=-q\left(2 U_{0}+C_{0}\right)+\left[2(2-q) g_{v}-q C_{1}\right] \mu, \quad \mu>0 .
$$

Левая часть уравнения (4.3) теперь определена на всей действительной оси. Правую часть уравнения (4.3) также нечетным образом продолжим на отрицательную полуось $\mu<0$. Получим уравнение, определенное на всей действительной оси:

$$
\int_{-\infty}^{\infty} \Phi(\eta, \mu) b(\eta) d \eta=-q\left(2 U_{0}+C_{0}\right) \operatorname{sign} \mu+\left[2(2-q) g_{v}-q C_{1}\right] \mu, \quad-\infty<\mu<\infty,
$$

где

$$
\operatorname{sign} x= \begin{cases}1, & x>0, \\ 0, & x=0, \\ -1, & x<0 .\end{cases}
$$

Подставляя в уравнение (4.4) функцию (2.7), получим сингулярное интегральное уравнение с ядром Коши

$$
\begin{aligned}
& \frac{1}{\sqrt{\pi}} \int_{-\infty}^{\infty} \frac{\eta b(\eta) d \eta}{\eta-\mu}+e^{\mu^{2}} \lambda(\mu) b(\mu)= \\
& \quad=-q\left(2 U_{0}+C_{0}\right) \operatorname{sign} \mu+\left[2(2-q) g_{v}-q C_{1}\right] \mu, \quad-\infty<\mu<\infty .
\end{aligned}
$$

Введем вспомогательную кусочно-аналитическую функцию

$$
M(z)=\frac{1}{\sqrt{\pi}} \int_{-\infty}^{\infty} \frac{\eta b(\eta) d \eta}{\eta-z},
$$


для которой

$$
M^{+}(\mu)-M^{-}(\mu)=2 \sqrt{\pi} i \mu b(\mu), \quad-\infty<\mu<\infty .
$$

С помошью граничных значений функций $M(z)$ и $\lambda(z)$ сведем уравнение (4.5) к краевой задаче определения аналитической функции по ее нулевому скачку на разрезе:

$$
\begin{gathered}
\lambda^{+}(\mu)\left\{M^{+}(\mu)-\left[2(2-q) g_{v}-q C_{1}\right] \mu\right\}-\lambda^{-}(\mu)\left\{M^{-}(\mu)-\left[2(2-q) g_{v}-q C_{1}\right] \mu\right\}= \\
=-2 \sqrt{\pi} i \mu e^{-\mu^{2}} \operatorname{sign} \mu\left(2 U_{0}+C_{0}\right) q, \quad-\infty<\mu<\infty .
\end{gathered}
$$

Задача (4.8) имеет следующее решение:

$$
M(z)=\left[2(2-q) g_{v}-q C_{1}\right] z-\left(2 U_{0}+C_{0}\right) q \frac{\psi_{1}(z)}{\lambda(z)}
$$

здесь

$$
\psi_{1}(z)=\frac{1}{\sqrt{\pi}} \int_{-\infty}^{\infty} \mu e^{-\mu^{2}} \operatorname{sign} \mu \frac{d \mu}{\mu-z} .
$$

Неизвестная функция $b(\eta)$ находится теперь из равенства (4.7), если в него подставить решение (4.9). Заметим, что

$$
\psi_{1}(z)=-\frac{1}{\sqrt{\pi} z}-\frac{1}{\sqrt{\pi} z^{3}}-\cdots-\frac{n !}{\sqrt{\pi} z^{2 n+1}}-\cdots, \quad z \rightarrow \infty .
$$

Воспользовавшись равенством из книги [10]

$$
\lambda(z)=-\frac{1}{2 z^{2}}-\frac{3}{4 z^{4}}-\cdots, \quad z \rightarrow \infty,
$$

получаем

$$
\frac{\psi_{1}(z)}{\lambda(z)}=\frac{2}{\sqrt{\pi}} z-\frac{1}{\sqrt{\pi} z}+\cdots, \quad z \rightarrow \infty .
$$

Устраним полюс в бесконечно удаленной точке у решения (4.9). С использованием асимптотического разложения (4.10) получаем уравнение

$$
2 U_{0} q+q C_{0}-\sqrt{\pi}(2-q) g_{v}+\frac{\sqrt{\pi}}{2} q C_{1}=0 .
$$

Уравнений (3.9) и (4.11) достаточно, чтобы решить задачу в нулевом приближении. Полагая в этих уравнениях $C_{1}=0$, находим, что в нулевом приближении

$$
\begin{aligned}
& U_{0}=\frac{2-q}{q}\left[V_{1} q+\frac{\sqrt{\pi}}{2}(1-q)\right] g_{v}, \\
& C_{0}=-2(2-q)\left(V_{1}-\frac{\sqrt{\pi}}{2}\right) g_{v} .
\end{aligned}
$$


Для реализации первого приближения требуется еще одно уравнение. Чтобы его получить, воспользуемся связью между решениями диффузной и зеркальной задач, которая непосредственно вытекает из условия (2.16) или из равенств (3.2) и (4.6):

$$
M(z)=N(z)-N(-z) .
$$

Подставим в (4.13) решения (3.7) и (4.9). Получаем

$$
\begin{aligned}
& {\left[2(2-q) g_{v}-q C_{1}\right] z-q\left(2 U_{0}+C_{0}\right) \frac{\psi_{1}(z)}{\lambda(z)}=} \\
& \quad=\left[4(2-q) g_{v}+2(1-q) C_{1}\right] z+d_{0}\left(\frac{1}{X(z)}-\frac{1}{X(-z)}\right) .
\end{aligned}
$$

Воспользовавшись далее асимптотическим разложением (3.8), находим

$$
\frac{1}{X(z)}-\frac{1}{X(-z)}=2 z+\frac{2 V_{1}^{*}}{z}+\frac{2 V_{3}^{*}}{z^{3}}+\cdots, \quad z \rightarrow \infty .
$$

Подставим разложения (4.10) и (4.15) в уравнение (4.14) и приравняем коэффициенты при $z$ и $z^{-1}$ в левой и правой частях этого уравнения. Получаем два уравнения:

$$
\begin{aligned}
2 U_{0} q+q C_{0}+\frac{\sqrt{\pi}}{2} q C_{1} & =\sqrt{\pi}(2-q) g_{v}, \\
2 U_{0} q+q C_{0}+2 \sqrt{\pi}(1-q) V_{1}^{*} C_{1} & =-4 \sqrt{\pi}(2-q) V_{1}^{*} g_{v} .
\end{aligned}
$$

Заметим, что уравнение (4.16) совпадает с уравнением (4.11). Это и понятно, ибо у функций $M(z)$ и $N(z)$ был уничтожен полюс первого порядка в бесконечно удаленной точке условиями

$$
\lim _{z \rightarrow \infty} \frac{M(z)}{z}=0, \quad \lim _{z \rightarrow \infty} \frac{N(z)}{z}=0 .
$$

Итак, в первом приближении для нахождения постоянных $U_{0}, C_{0}, C_{1}$ требуется решить систему уравнений (3.9), (4.11) и (4.17). Приведем выражение для скорости изотермического скольжения в стандартном виде: $U_{0}=K_{v}(q) g_{v}$, где $K_{v}(q)$ - коэффициент изотермического скольжения,

$$
K_{v}(q)=\frac{2-q}{q}\left[V_{1}+2 V_{1}^{*} \frac{2 V_{1}-\sqrt{\pi}}{q-4 V_{1}^{*}(1-q)}(1-q)\right] .
$$

В табл. 1 столбец I содержит значения коэффициента изотермического скольжения, рассчитанные модифицированным методом дискретных ординат [8], столбец IV - данные из работы [7], а в столбцах II (нулевое приближение) и III (первое приближение) приведены данные настояшей работы. 
ТАБЛИцА 1

\begin{tabular}{|c|c|c|c|c|}
\hline $\mathrm{q}$ & $\mathrm{I}$ & $\mathrm{II}$ & $\mathrm{III}$ & $\mathrm{IV}$ \\
\hline 0.1 & 17.10313 & 17.08524 & 17.10066 & 17.09809 \\
0.2 & 8.224902 & 8.209878 & 8.222870 & 8.220481 \\
0.3 & 5.255112 & 5.242892 & 5.253472 & 5.251263 \\
0.4 & 3.762619 & 3.752850 & 3.761328 & 3.759290 \\
0.5 & 2.861190 & 2.853627 & 2.860205 & 2.858334 \\
0.6 & 2.255410 & 2.249813 & 2.254692 & 2.252980 \\
0.7 & 1.818667 & 1.814803 & 1.818179 & 1.816621 \\
0.8 & 1.487654 & 1.485297 & 1.487361 & 1.485952 \\
0.9 & 1.227198 & 1.226127 & 1.227066 & 1.225801 \\
1.0 & 1.016191 & 1.016191 & 1.016191 & 1.015064 \\
\hline
\end{tabular}

Из формул (4.12) и (4.18) видно, что $K_{v}(q)=\sqrt{\pi} / q$ при $q \rightarrow 0$; это в точности совпадает с известным результатом Черчиньяни [11].

Анализ данных из табл. 1 показывает, что уже в нулевом приближении разработанный метод обеспечивает точность вычисления величины $K_{v}$ не менее одного процента во всем диапазоне изменения величины $q$. А в первом приближении из анализа данных этой таблицы видно, что при $q=0.1$ различие коэффициента скольжения, рассчитанного в данной работе, и коэффициента, рассчитанного с применением численных методов, составляет $0.014 \%$, при $q=0.5-0.034 \%$, при $q=0.9-0.087 \%$; при $q=1$ результаты совпадают.

\section{5. ЗАДАЧА О ТЕПЛОВОМ СКОЛЬЖЕНИИ}

Как известно [10]-[13], задача о тепловом скольжении с использованием уравнения Бхатнагара-Гросса-Крука (БГК) сводится к решению следующей граничной задачи:

$$
\begin{aligned}
& \mu \frac{\partial \psi}{\partial x}+\psi(x, \mu)+g_{T}\left(\mu^{2}-\frac{1}{2}\right)=\frac{1}{\sqrt{\pi}} \int_{-\infty}^{\infty} e^{-\mu^{\prime 2}} \psi\left(x, \mu^{\prime}\right) d \mu^{\prime}, \\
& \psi(0, \mu)=(1-q) \psi(0,-\mu), \quad \mu>0, \\
& \psi(x, \mu)=\psi_{\mathrm{as}}(\mu)+o(1), \quad x \rightarrow+\infty, \quad \mu<0 .
\end{aligned}
$$

Здесь

$$
\psi_{\text {as }}(\mu)=2 U_{0}-g_{T}\left(\mu^{2}-\frac{1}{2}\right),
$$

$g_{T}$ - градиент логарифма температуры, заданный вдали от стенки.

Решение задачи (5.1)-(5.3) будем искать в виде $\psi(x, \mu)=\psi_{\mathrm{as}}(x, \mu)+\psi_{\mathrm{c}}(x, \mu)$, где $\psi_{\mathrm{c}}(x, \mu)$ определяется равенством $(2.9)$. 
Перепишем условие (5.2) для функции $\psi_{\mathrm{c}}$ :

$$
\psi_{\mathrm{c}}(0, \mu)-(1-q) \psi_{\mathrm{c}}(0,-\mu)=\psi_{0}(\mu)
$$

где

$$
\psi_{0}(\mu)=-2 U_{0} q+q\left(\mu^{2}-\frac{1}{2}\right) g_{T} .
$$

Рассуждая, как и выше, получаем диффузное и зеркальное граничные условия:

$$
\begin{aligned}
& \psi_{\mathrm{c}}(0, \mu)=\psi_{0}(\mu)+(1-q) C(\mu), \quad \mu>0 \\
& \psi_{\mathrm{c}}(0, \mu)-\psi_{\mathrm{c}}(0,-\mu)=\psi_{0}(\mu)-q C(\mu), \quad \mu>0
\end{aligned}
$$

Условие вдали от стенки здесь прежнее: $\psi_{\mathrm{c}}(\infty, \mu)=0$.

Решение задачи с диффузным условием (5.4) приводит к следующей краевой задаче:

$$
X^{+}(\mu)\left[N^{+}(\mu)+2 U_{0} q-q\left(\mu^{2}-\frac{1}{2}\right) g_{T}\right]=X^{-}(\mu)\left[N^{-}(\mu)+2 U_{0} q-q\left(\mu^{2}-\frac{1}{2}\right) g_{T}\right], \quad \mu>0 .
$$

Здесь функция $N(z)$ определяется также выражением (3.2), а функция $X(z)$ введена выше.

Общее решение задачи (5.6) имеет вид

$$
N(z)=-2 U_{0} q+q\left(z^{2}-\frac{1}{2}\right) g_{T}+(1-q)\left(C_{0}+C_{1} z\right)+\frac{d_{0}+d_{1} z}{X(z)}
$$

где $d_{0}, d_{1}-$ произвольные постоянные.

Решение (5.7) имеет в бесконечно удаленной точке полюс второго порядка. Для устранения этого полюса воспользуемся разложением (3.8). Подставим (3.8) в (5.7) и приравняем нулю коэффициенты при $z^{k}, k=0,1,2$. Получаем три уравнения: $d_{1}=-q g_{T}$, $d_{0}=-(1-q) C_{1}-q V_{1} g_{T}$,

$$
-2 U_{0} q+(1-q)\left(C_{0}+C_{1} V_{1}\right)=-2 q K_{T}^{\circ} g_{T}
$$

где $K_{T}^{\circ}$ - коэффициент теплового скольжения при $q=1$ [10], [12],

$$
K_{T}^{\circ}=\frac{1}{2}\left(V_{2}+\frac{1}{2} V_{1}^{2}-\frac{1}{2}\right)=0.383161 \text {. }
$$

Решая задачу с зеркальным условием (5.5), приходим к краевой задаче

$$
\begin{aligned}
\lambda^{+}(\mu)[ & \left.M^{+}(\mu)+q C_{1} \mu\right]-\lambda^{-}(\mu)\left[M^{-}(\mu)+q C_{1} \mu\right]= \\
& =2 \sqrt{\pi} i \mu e^{-\mu^{2}} \operatorname{sign} \mu\left[-\left(2 U_{0}+C_{0}\right) q+q\left(\mu^{2}-\frac{1}{2}\right) g_{T}\right], \quad-\infty<\mu<\infty
\end{aligned}
$$

где функция $M(z)$ введена равенством (4.6). 
Эта задача имеет решение

$$
M(z)=-q C_{1} z+q g_{T} \frac{\psi_{3}(z)}{\lambda(z)}-q\left(2 U_{0}+C_{0}+\frac{g_{T}}{2}\right) \frac{\psi_{1}(z)}{\lambda(z)} .
$$

Здесь функция $\psi_{1}(z)$ была введена ранее, а

$$
\psi_{3}(z)=\frac{1}{\sqrt{\pi}} \int_{-\infty}^{\infty} \mu^{3} e^{-\mu^{2}} \frac{\operatorname{sign} \mu}{\mu-z} d \mu
$$

Эта функция в окрестности бесконечно удаленной точки разлагается в асимптотический ряд

$$
\psi_{3}(z)=-\frac{1}{\sqrt{\pi} z}-\frac{2}{\sqrt{\pi} z^{3}}-\cdots-\frac{(n+1) !}{\sqrt{\pi} z^{2 n+1}}-\cdots, \quad z \rightarrow \infty .
$$

Следовательно, отношение $\psi_{3} / \lambda$ разлагается в асимптотический ряд

$$
\frac{\psi_{3}(z)}{\lambda(z)}=\frac{2}{\sqrt{\pi}} z+\frac{1}{\sqrt{\pi} z}+\cdots, \quad z \rightarrow \infty
$$

Подставив его в $(5.9)$, при условии $M(\infty)=0$ получаем уравнение

$$
2 U_{0} q+q C_{0}+\frac{\sqrt{\pi}}{2} q C_{1}=\frac{1}{2} q g_{T} .
$$

Подставив функцию $N(z)$ в условие $(4.13)$, получим для функции $M(z)$ разложение

$$
\begin{aligned}
M(z)= & 2(1-q) C_{1} z+d_{0}\left(\frac{1}{X(z)}-\frac{1}{X(-z)}\right)+ \\
& +d_{1} z\left(\frac{1}{X(z)}+\frac{1}{X(-z)}\right)
\end{aligned}
$$

Воспользуемся разложением (4.15) и разложением

$$
\frac{1}{X(z)}+\frac{1}{X(-z)}=\frac{2}{z}\left(-V_{1} z+\frac{V_{2}^{*}}{z}+\frac{V_{4}^{*}}{z^{3}}+\cdots\right), \quad z \rightarrow \infty
$$

Теперь получаем, что функция $M(z)$, определенная равенством (5.11), разлагается в асимптотический ряд

$$
M(z)=2\left(d_{0} V_{1}^{*}+D_{1} V_{2}^{*}\right) \frac{1}{z}+\cdots
$$

С другой стороны, решение (5.9) также можно разложить в асимптотический ряд:

$$
M(z)=\left(2 U_{0} q+q C_{0}+\frac{3}{2} q g_{T}\right) \frac{1}{\sqrt{\pi} z}+\cdots .
$$

Сравнивая разложения (5.12) и (5.13), получаем третье уравнение для определения $U_{0}, C_{0}, C_{1}$ :

$$
2 U_{0} q+q C_{0}+\frac{3}{2} q g_{T}=2 \sqrt{\pi}\left(V_{1}^{*} d_{0}+V_{2}^{*} d_{1}\right)
$$


Подставляя в это равенство найденные выше значения $d_{0}, d_{1}$, окончательно получаем

$$
2 U_{0} q+q C_{0}+2 \sqrt{\pi} V_{1}^{*}(1-q) C_{1}=-q\left[\frac{3}{2}+2 \sqrt{\pi}\left(V_{1} V_{1}^{*}+V_{2}\right)\right] g_{T}
$$

Рассмотрим сначала нулевое приближение задачи. Из уравнений (5.8) и (5.10) при $C_{1}=0$ находим $U_{0}=K_{T}^{(0)}(q) g_{T}$, где

$$
K_{T}^{(0)}(q)=q K_{T}^{\circ}+0.25(1-q)
$$

Из формулы (5.15) при $q=1$ получаем известный результат [10], [12]: $K_{T}^{(0)}(1)=K_{T}^{\circ}$, а при $q=0$ - известный результат Максвелла: $K_{T}^{(0)}(0)=0.25$.

Рассмотрим первое приближение. Из системы уравнений (5.8), (5.10) и (5.14) находим $U_{0}, C_{0}, C_{1}$. Выпишем формулу для нахождения скорости теплового скольжения: $U_{0}=K_{T}^{(1)}(q) g_{T}$, где коэффициент теплового скольжения определяется равенством

$$
K_{T}^{(1)}(q)=K_{T}^{(0)}(q)+\gamma \frac{q(1-q)}{q-4 V_{1}^{*}(1-q)}
$$

при

$$
\gamma=\frac{2}{\sqrt{\pi}}\left(V_{1}-\frac{\sqrt{\pi}}{2}\right)\left(1+\sqrt{\pi} V_{1} V_{1}^{*}+\sqrt{\pi} V_{2}^{*}\right)=0.008609 .
$$

Приведем табл. 2, в которой столбец I содержит данные из работы [8], полученные с помошью численных методов, столбцы II и III - данные настояшей работы, рассчитанные по формулам нулевого и первого приближений (5.15) и (5.16), в столбце IV приведены данные из работы [7].

ТАБЛИЦА 2

\begin{tabular}{|c|c|c|c|c|}
\hline $\mathrm{q}$ & $\mathrm{I}$ & $\mathrm{II}$ & $\mathrm{III}$ & $\mathrm{IV}$ \\
\hline 0.1 & 0.2641783 & 0.2633161 & 0.2641393 & 0.263956 \\
0.2 & 0.2781510 & 0.2766322 & 0.2780850 & 0.277741 \\
0.3 & 0.2919238 & 0.2899483 & 0.2918429 & 0.291357 \\
0.4 & 0.3055019 & 0.3032644 & 0.3054149 & 0.304807 \\
0.5 & 0.3188906 & 0.3165805 & 0.3188055 & 0.318096 \\
0.6 & 0.3320949 & 0.3298976 & 0.3320183 & 0.331227 \\
0.7 & 0.3451195 & 0.3432127 & 0.3450568 & 0.344202 \\
0.8 & 0.3579692 & 0.3565298 & 0.3579245 & 0.357024 \\
0.9 & 0.3706483 & 0.3698449 & 0.3706248 & 0.369697 \\
1.0 & 0.3831612 & 0.3831612 & 0.3831612 & 0.382223 \\
\hline
\end{tabular}


Анализ данных из табл. 2 показывает, что уже в нулевом приближении разработанньй метод обеспечивает точность не менее одного процента во всем диапазоне изменения величины $q$. А в первом приближении результаты настоящей работы точнее соответствующих результатов из статьи [7]. При $q=0.1$ точность первого приближения составляет $0.015 \%$, при $q=0.5-0.028 \%$, при $q=0.9-0.006 \%$.

\section{6. ЗАДАЧА КРАМЕРСА ДЛЯ УРАВНЕНИЯ ВИЛЬЯМСА}

Как известно [13], задача об изотермическом скольжении с использованием уравнения Вильямса состоит в решении следуюшей граничной задачи:

$$
\begin{aligned}
& \mu \frac{\partial \psi}{\partial x}+\psi(x, \mu)=\frac{3}{4} \int_{-1}^{1}\left(1-\mu^{\prime 2}\right) \psi\left(x, \mu^{\prime}\right) d \mu^{\prime}, \\
& \psi(0, \mu)=(1-q) \psi(0,-\mu), \quad 0<\mu<1, \\
& \psi(x, \mu)=\psi_{\mathrm{as}}(x, \mu)+o(1), \quad x \rightarrow+\infty, \quad-1<\mu<0,
\end{aligned}
$$

где $\psi_{\text {as }}(x, \mu)=2 U_{0}+2 g_{v}(x-\mu)$.

Для решения задачи (6.1)-(6.3) представим функцию $\psi$ в виде суммы: $\psi(x, \mu)=$ $\psi_{\mathrm{as}}(x, \mu)+\psi_{\mathrm{c}}(x, \mu)$. Перепишем граничное условие $(6.2)$ для функции $\psi_{\mathrm{c}}$ :

$$
\psi_{\mathrm{c}}(0, \mu)-(1-q) \psi_{\mathrm{c}}(0,-\mu)=\psi_{0}(\mu), \quad 0<\mu<1 \text {, }
$$

где $\psi_{0}(\mu)=-2 U_{0} q+2 g_{v}(2-q) \mu$. Как и ранее, заменим условие (6.4) двумя:

$$
\begin{aligned}
& \psi_{\mathrm{c}}(0, \mu)=\psi_{0}(\mu)+(1-q) C(\mu), \quad 0<\mu<1, \\
& \psi_{\mathrm{c}}(0, \mu)-\psi_{\mathrm{c}}(0,-\mu)=\psi_{0}(\mu)-q C(\mu), \quad 0<\mu<1 .
\end{aligned}
$$

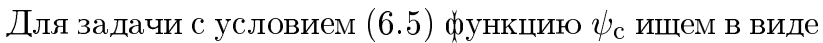

$$
\psi_{\mathrm{c}}(x, \mu)=\int_{0}^{1} e^{-x / \eta} \Phi(\eta, \mu) a(\eta) d \eta
$$

а для задачи с условием (6.6) - в виде

$$
\psi_{\mathrm{c}}(x, \mu)=\int_{0}^{1} e^{-x / \eta} \Phi(\eta, \mu) b(\eta) d \eta
$$

Здесь $\Phi(\eta, \mu)$ - собственная функция характеристического уравнения Вильямса [13]:

$$
\Phi(\eta, \mu)=\frac{3}{4} \eta P \frac{1}{\eta-\mu}+\frac{\lambda_{\mathrm{w}}(\eta)}{1-\eta^{2}} \delta(\eta-\mu),
$$

$\lambda_{\mathrm{w}}(z)$ - дисперсионная функция Вильямса,

$$
\lambda_{\mathrm{w}}(z)=1+\frac{3}{4} \int_{0}^{1} \frac{1-\tau^{2}}{\tau-z} d \tau=-\frac{1}{2}+\frac{3}{2}\left(1-z^{2}\right) \lambda(z),
$$


$\lambda(z)$ - дисперсионная функция Кейза [14],

$$
\lambda(z)=1+\frac{z}{2} \int_{-1}^{1} \frac{d \tau}{\tau-z} .
$$

Решение задачи с диффузным условием (6.5) на стенке приводит в первом приближении к функции

$$
N(z)=-2 U_{0} q+2 g_{v}(2-q) z+(1-q)\left(C_{0}+C_{1} z\right)+\frac{d}{X(z)},
$$

причем

$$
N(z)=\frac{3}{4} \int_{0}^{1} \frac{\eta a(\eta) d \eta}{\eta-z}, \quad d=-2(2-q) g_{v}-(1-q) C_{1} .
$$

Здесь

$$
\begin{gathered}
X(z)=\frac{1}{z} e^{V(z)}, \quad V(z)=\frac{1}{\pi} \int_{0}^{1} \frac{\zeta_{\mathrm{w}}(\tau) d \tau}{\tau-z}, \\
\zeta_{\mathrm{w}}(\tau)=-\frac{\pi}{2}-\operatorname{arctg} \frac{4 \lambda_{\mathrm{w}}(\tau)}{3 \pi \tau\left(1-\tau^{2}\right)} .
\end{gathered}
$$

Условие разрешимости в этом случае приводит к уравнению

$$
-2 U_{0} q+(1-q)\left(C_{0}+C_{1} V_{1}\right)+2(2-q) V_{1} g_{v}=0
$$

где

$$
V_{k}=-\frac{1}{\pi} \int_{0}^{1} \zeta_{\mathrm{w}}(\tau) \tau^{k-1} d \tau, \quad k=1,2, \ldots, \quad V_{1}=0.581946, \quad V_{2}=0.214286 .
$$

Решение задачи с зеркальным условием (6.6) приводит к функции

$$
M(z)=\left[2(2-q) g_{v}-q C_{1}\right] z-\left(2 U_{0}+C_{0}\right) q \frac{\psi(z)}{\lambda_{\mathrm{w}}(z)} .
$$

Здесь

$$
\psi(z)=\frac{3}{4} \int_{0}^{1} \frac{\mu\left(1-\mu^{2}\right) \operatorname{sign} \mu}{\mu-z} d \mu=-\frac{3}{8 z}-\frac{1}{8 z^{3}}-\frac{1}{16 z^{5}}-\cdots, \quad|z|>1 .
$$

Подставляя разложение

$$
\frac{\psi(z)}{\lambda_{\mathrm{w}}(z)}=\frac{15}{8} z-\frac{5}{28 z}+\cdots,|z|>1,
$$

в (6.9) и устраняя полюс в бесконечно удаленной точке, получаем уравнение

$$
2 U_{0} q+q C_{0}+\frac{8}{15} q C_{1}=\frac{16}{15}(2-q) g_{v} .
$$


Функции $M(z), N(z)$ по-прежнему связаны условием (4.13). Подставляя в это условие (6.7) и (6.9), разложим полученное равенство в ряд Лорана в окрестности бесконечно удаленной точки и приравняем коэффициенты при $z^{-1}$ слева и справа. Приходим к уравнению

$$
2 U_{0} q+q C_{0}+\frac{56}{5} V_{1}^{*}(1-q) C_{1}=-\frac{112}{5} V_{1}^{*}(2-q) g_{v},
$$

где

$$
V_{1}^{*}=\frac{1}{2} V_{1}^{2}-V_{2}=-0.044955 .
$$

Из системы уравнений $(6.8),(6.10)$ и (6.11) находим неизвестные параметры $U_{0}, C_{0}$, $C_{1}$

Рассмотрим нулевое приближение. Из системы уравнений (6.8) и (6.10) при $C_{1}=0$ находим скорость изотермического скольжения:

$$
U_{0}=\frac{2-q}{q}\left[V_{1} q+\frac{8}{15}(1-q)\right] g_{v}
$$

Из этой формулыпри $q=1$ получаем $U_{0}=V_{1} g_{v}-$ известный [10] результат, а при $q \rightarrow$ 0 получаем $U_{0}=16 /(15 q)$, что также совпадает с известным результатом из книги [10].

Рассмотрим первое приближение. В этом случае из системы уравнений (6.8), (6.10) и (6.11) находим скорость изотермического скольжения $U_{0}=K_{v}^{(1)}(q) g_{v}$, где

$$
K_{v}^{(1)}(q)=\frac{2-q}{q}\left[V_{1}+(1-q) \frac{21 V_{1} V_{1}^{*}-11.2 V_{1}^{*}}{q-21 V_{1}^{*}(1-q)}\right] .
$$

Формулы для скорости изотермического скольжения приведем к размерному виду. Рассуждая так же, как и в книге [13], для размерной скорости $u_{\mathrm{sl}}$ получаем, что $u_{\mathrm{sl}}=$ $K_{v}^{*}(q) l g_{v}^{*}, l$ - длина свободного пробега молекул [11], $g_{v}^{*}$ - градиент размерной массовой скорости, причем в случае БГК-уравнения

$$
K_{v}^{*}(q)=\frac{2}{\sqrt{\pi}} K_{v}(q)
$$

где коэффициент $K_{v}(q)$ определяется согласно (4.18), а в случае уравнения Вильямса

$$
K_{v}^{*}(q)=\frac{15}{8} K_{v}(q)
$$

где $K_{v}(q)$ определяется согласно (6.13).

Приведем в табл. 3 значения коэффициента изотермического скольжения, рассчитанные по формулам (6.12) и (6.13). Столбец I содержит данные нулевого приближения, столбец II - данные первого приближения, а в столбцах III и IV приведены данные коэффициента размерной скорости скольжения, при этом столбец III содержит данные, рассчитанные по формуле (6.15), а столбец IV - по формуле (6.14). 
ТАБЛИЦА 3

\begin{tabular}{|c|c|c|c|c|}
\hline $\mathrm{q}$ & $\mathrm{I}$ & $\mathrm{II}$ & $\mathrm{III}$ & $\mathrm{IV}$ \\
\hline 0.1 & 10.22570 & 10.230595 & 19.182385 & 19.29603 \\
0.2 & 4.887503 & 4.891603 & 9.171755 & 9.278515 \\
0.3 & 3.104864 & 3.108232 & 5.827935 & 5.927909 \\
0.4 & 2.211114 & 2.213815 & 4.105903 & 4.244204 \\
0.5 & 1.672919 & 1.675017 & 3.140658 & 3.227396 \\
0.6 & 1.312502 & 1.314060 & 2.463863 & 2.544147 \\
0.7 & 1.053873 & 1.054751 & 1.977659 & 2.051595 \\
0.8 & 0.858335 & 0.858995 & 1.610616 & 1.678307 \\
0.9 & 0.705326 & 0.705627 & 1.323050 & 1.384596 \\
1.0 & 0.581946 & 0.581946 & 1.091149 & 1.146649 \\
\hline
\end{tabular}

Данные столбцов III и IV показывают, что значения коэффициента изотермического скольжения для газов с постоянной частотой столкновений молекул и для газов с частотой столкновений, пропорциональной модулю скорости молекул, достаточно близки друг другу. Так, наибольшее отличие этих коэффициентов составляет $4.8 \%$ при $q=1$, при $q=0.5$ отличие равно $2.7 \%$, а при $q=0.1-0.6 \%$. При $q \rightarrow 0$ значения коэффициентов совпадают (отличие между ними стремится к нулю при $q \rightarrow 0$ ), что вытекает из полученных асимптотик.

\section{7. ОБСУЖ ДЕНИЕ РЕЗУЛЬТАТОВ. ВЫВОДЫ И ЗАКЛЮЧЕНИЕ}

Сначала опишем кратко метод аналитического решения граничной задачи для кинетического уравнения, например, в случае диффузного отражения молекул от стенки.

Разделение переменных в кинетическом уравнении приводит к характеристическому уравнению. Находятся собственные функции и спектр (непрерьвный и дискретньй) последнего, а также собственные решения исходного кинетического уравнения. Составляется обшее решение граничной задачи, представляющее собой сумму линейной комбинации дискретных решений и интеграла по непрерывному спектру. Этот интеграл есть интеграл по действительной полуоси в случае постоянной частоты молекул и по отрезку $[0,1]$ в случае частоты столкновений, пропоршиональной модулю скорости молекул. Интеграл по непрерывному спектру вычисляется от произведения собственной функции характеристического уравнения для неизвестной функции, называемой коэффициентом непрерывного спектра.

Разложение общего решения после подстановки в граничное условие на стенке сводится к сингулярному интегральному уравнению с ядром Коши. Основные трудности приходятся на решение этого уравнения. 
Предположим теперь, что решается граничная задача с зеркально-диффузным условием Максвелла на стенке (с коэффициентом аккомодации $q$, или, что все равно, с коэффициентом зеркальности $1-q)$.

В этом случае в получаемом сингулярном интегральном уравнении два ядра, одно из которых имеет прямой сдвиг, а второе - обратный сдвиг (разностное и сумматорное ядра). Это уравнение решить аналитически не представляется возможным. Методы решения таких уравнений отсутствуют и в литературе [15]. Чтобы решить это сингулярное уравнение, предлагается следующий метод.

Суть метода основана на представлении условия Максвелла для суммы двух линейно независимых условий. Одно из таких условий является зеркальным, а другое - диффузным.

Такое представление условия Максвелла является вполне естественным. В самом деле, условие Максвелла, объединяя чисто зеркальное и чисто диффузное условие, записывается для отраженных от стенки молекул. Этим нарушается симметрия чисто зеркального условия. В чисто зеркальном условии сушествует инвариантность относительно замены летящих к стенке молекул отраженными. При этом функция распределения летящих к стенке молекул переходит в функцию распределения отраженных от стенки молекул.

Итак, после замены условия Максвелла двумя - зеркальным и диффузным - исходная граничная задача эквивалентна двум задачам, допускаюшим аналитическое решение. Это принципиальный момент метода. Обе эти задачи записьваются для части функции распределения $\psi_{\mathrm{c}}(x, \mu)$, отвечающей непрерьвному спектру.

При переходе от исходной граничной задачи к двум в граничных условиях последних появляется неизвестная функция $C(\mu)$, которая заменяется аппроксимационным многочленом $C_{n}(\mu) n$-й степени. Коэффициенты этого многочлена находятся из системы линейных уравнений, первое из которых есть условие разрешимости соответствуюшей краевой задачи Римана. Остальные уравнения выводятся следуюшим образом. Воспользуемся связью (2.16) между решениями граничных задач, что приводит к соотношению (4.13) между решениями краевых задач Римана из диффузной и зеркальной задач. Подставляя в (4.13) соответствуюшие полученные решения и приравнивая коэффициенты при одинаковых степенях $z$, получаем недостающие уравнения системы для определения коэффициентов многочлена $C_{n}(z)$. Увеличивая неограниченно степень многочлена $C_{n}(z)$, в пределе при $n \rightarrow \infty$ получаем точное выражение функции $C(z)$, а следовательно, и точное решение задачи. В этом смысле предлагаемый метод является регулярным. Повышая степень многочлена $C_{n}(z)$, можно получить решение с любой заданной степенью точности.

Действуя методом, предложенным в работе [7], также можно получить решение граничной задачи с любой заданной степенью точности. Этот метод особенно удобен для решения тех классов граничных задач, в которых разложение решения по собственным функциям сводится к решению краевой задачи Римана, для коэффициента которого решить задачу факторизации аналитически не представляется возможным. Однако преимушество метода, предлагаемого в данной работе, состоит в том, что уже в первом 
приближении он дает бо́льшую точность, нежели метод из работы [7].

В заключение отметим, что изложенный метод может быть применен в самых разнообразных граничных задачах кинетической теории - в теории переноса нейтронов, в теории кристаллитов, при описании поведения плазмы, при решении задач теории скин-эффекта, при решении задач о температурном скачке в газе, в металле, при описании взаимодействия электромагнитного поля с металлом и т.д.

Благодарности. Работа выполнена при частичной (А. В. Латышев) финансовой поддержке РФФИ (грант № 03-01-00281).

\section{Список литературы}

[1] И. К. Лифанов. Метод сингулярных интегральных уравнений и численный эксперимент (в математической физике, аэродинамике, теории упругости и дифракции волн). М.: ТОО "Янус", 1995.

[2] K. Черчиньяни. Теория и приложения уравнения Больцмана. М.: Мир, 1978.

[3] В. В. Веденяпин. Кинетические уравнения Больцмана и Власова. М.: Физматлит, 2001; W. Greenberg, C. van der Mee, V. Protopopescu. Boundary Value Problems in Abstract Kinetic Theory. Basel: Birkhäuser, 1987.

[4] А. В. Латыщев, А. А. Юиканов. Изв. РАН. Сер. МЖГ. 1996. № 3. С. 140.

[5] А. В. Латыиев, А. А. Юиканов. ЖВМиМФ. 1997. Т. 37. № 4. С. 483.

[6] А. В. Латышев, А. А. Юиканов. ТМФ. 1997. Т. 111. № 3. С. 462; ЖТФ. 1998. Т. 68. № 11. C. 27 .

[7] А. В. Латыиев, А. А. Юиканов. ЖВМиМФ. 2004. Т. 44. № 6. С. 1107.

[8] C. E. Siewert, F. Sharipov. Phys. Fluids. 2002. V. 14. № 12. P. 4123.

[9] В. С. Владимиров, В. В. ЖКаринов. Уравнения математической физики. М.: Физматлит, 2000.

[10] A. В. Латышев, А. А. Юшканов. Аналитическое решение граничных задач кинетической теории. М.: Изд-во МГОУ, 2004.

[11] K. Черчиньяни. Математические методы в кинетической теории газов. М.: Мир, 1973.

[12] S. K. Loyalka, J. W. Cipolla. Phys. Fluids. 1971. V. 14. № 8. P. 1656.

[13] А. В. Латышев, А. А. Юшканов. Кинетические уравнения типа Вильямса и их точные решения. М.: Изд-во МГОУ, 2004.

[14] К. Кейз, П. Цвайфель. Линейная теория переноса. М.: Мир, 1972.

[15] Г. С. Литвинчук. Краевые задачи и сингулярные интегральные уравнения со сдвигом. М.: Наука, 1977.

Поступила в редакцию 28.IX.2004 г., после доработки 26.XI.2004 г. 